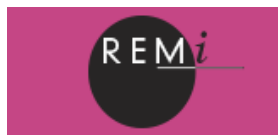

Revue européenne des migrations internationales

vol. 28 - n³ | 2012

La mort en migration

\title{
Éditorial : La mort en migration
}

Editorial: Death in Migration

Editorial: La muerte en migración

\section{Françoise Lestage}

\section{(2) OpenEdition}

\section{Journals}

Édition électronique

URL : https://journals.openedition.org/remi/5992

DOI : $10.4000 /$ remi.5992

ISSN : $1777-5418$

Éditeur

Université de Poitiers

Édition imprimée

Date de publication : 1 septembre 2012

Pagination : 7-12

ISBN : 979-10-90426-05-4

ISSN : 0765-0752

Référence électronique

Françoise Lestage, «Éditorial : La mort en migration », Revue européenne des migrations internationales

[En ligne], vol. 28 - n³ | 2012, mis en ligne le 01 septembre 2015, consulté le 15 avril 2022. URL

http://journals.openedition.org/remi/5992 ; DOI : https://doi.org/10.4000/remi.5992 


\section{Éditorial}

\section{La mort en migration}

\section{Françoise Lestage ${ }^{1}$}

Ce dossier se propose de fournir des éléments, notamment ethnographiques, pour documenter et interroger la mort en migration, un sujet peu traité par les sciences sociales. II s'agira notamment de réfléchir à la constitution de territoires des morts d'origine étrangère dans la cité, à la faveur de certains événements historiques (guerres mondiales ou civiles); des territoires évolutifs en fonction des mutations de la migration (célibataires puis familiales) ; des territoires circulatoires, englobant le rapatriement des corps, qui alimentent l'activité d'entreprises spécialisées (transports et entreprises funéraires) et bouclent le cycle de vie par le retour au lieu de naissance. Les quatre articles du dossier présentent et analysent les pratiques de migrants originaires d'aires géographiques et socio-culturelles variées (Asie, Afrique, Amérique) à I'occasion du décès d'un des leurs. II ne s'agit pas ici d'élaborer des théories, mais d'inscrire ces pratiques dans des questionnements relatifs aux migrations.

De loin en loin, la mort en migration a suscité quelques travaux et ce dossier s'inscrit dans leur prolongement. En France, dans les années 1980 et 1990, des pionniers ont ouvert la voie (Branquinho Pequeno, 1983 ; Catani, 1988 ; Trong Hien Dinh, 1990), creusée par d'autres chercheurs dans les années 2000 (Chaïb, 2000 ; Petit, 2002 et 2005 ; Pourette, 2002 ; Aggoun, 2006 et 2009), chacun traitant de migrants originaires de lieux différents : Europe, Asie, Maghreb, départements d'outre-mer. Dans l'ouvrage de Yassine Chaïb (2000), L'émigré et la mort, l'auteur part de l'étude des transferts de corps de la France vers la Tunisie pour s'interroger sur les " routes de la mort ", les causes des décès des migrants ou le poids des emplacements des sépultures dans l'appartenance familiale et nationale. Ce livre, riche en pistes de recherche, a inspiré les travaux qui ont suivi, et tous les auteurs de ce dossier y font référence. Au même moment, Agathe Petit s'attache à l'étude des comportements des migrants ouest-africains qui rapatrient les défunts grâce à des associations, les "caisses des morts ", dont l'activité principale est le financement des transferts des corps vers le pays d'origine (Petit, 2002), et sur laquelle reviennent Clara Saravia et José Mapril dans ce numéro. Toujours dans la décennie des années 2000, Atmane Aggoun, après s'être penché sur le décès des migrants musulmans âgés en France (Aggoun, 2006), analyse la construction d'une mémoire et d'une histoire musulmane prenant appui sur la patrimonialisation du cimetière franco-musulman de Bobigny (Aggoun, 2009), cimetière qui fait aussi I'objet de I'article de Soraya El Alaoui dans ce dossier.

1 Professeur d'anthropologie, Université Paris Diderot, Laboratoire URMIS (UMR 205), case courrier 7027, 75205 Paris cedex 13 ; francoise.lestage@univ-paris-diderot.fr 
Ailleurs, la mort en migration constitue également une piste de recherche féconde : celle des musulmans (Fall et Ndongo Dime, 2011), celle des Portoricains à New York (Berthod, 2006) ou des Mexicains aux États-Unis (Lestage, 2008 et 2009 ; Felix, 2011), cette dernière également abordée dans ce dossier. II convient enfin de replacer l'ensemble de ces travaux au croisement des études sur les migrations et d'un vaste champ des sciences sociales portant sur la mort, qui a ses chercheurs (Philippe Ariès, Patrick Baudry, Maurice Bloch, Jean-Hugues Déchaux, William Douglass, Norbert Elias, Robert Hertz, ou Louis-Vincent Thomas pour ne citer que quelques-uns parmi les plus connus), ses revues (Études sur la mort ; Frontières) et d'innombrables publications.

\section{L'État et les restes humains}

Les États ont un rôle de premier plan dans le traitement des restes humains parce qu'ils les contrôlent et " les assignent à des espaces délimités " (Esquerre et Truc, 2011 : 10), espaces publics dans la France contemporaine. Face à la mort des migrants, les pays d'accueil sont donc amenés à élaborer des politiques publiques concernant des espaces funéraires destinés à des défunts dont la religion exige des conditions spécifiques pour leurs sépultures, tout en conservant la laïcité de ces lieux, inscrite dans le droit.

Deux articles de ce dossier traitent de la création et de la gestion des espaces funéraires musulmans dans des pays majoritairement catholiques. Soraya El Alaoui rappelle comment l'État français a créé un cimetière musulman dans les années 1930, pour des morts aux profils sociaux, nationaux et religieux très divers. Produit de représentations syncrétiques de l'espace funéraire élaborées par les fonctionnaires musulmans qui l'ont administré pendant plus d'un quart de siècle, ce cimetière sert aujourd'hui de " lieu de mémoire " pour bâtir l'histoire des musulmans de France (Aggoun, 2009). Jordi Moreras et Sol Tarrés font un inventaire des carrés et cimetières musulmans en Espagne en montrant clairement que ce qui semble être une " nouvelle " entreprise politique et administrative ne fait que relancer une réflexion déjà entamée, et résolue, à l'époque de la colonisation et des guerres impliquant des musulmans dans l'Espagne de la première moitié du XXe siècle.

Ces deux articles soulignent notamment comment les morts " participent à la construction et à la définition des collectifs humains "(Bondaz, 2011) en mettant en évidence les liens entre les morts musulmans d'aujourd'hui, en France comme en Espagne, et ceux de I'histoire coloniale des deux pays par le biais des cimetières adjacents aux hôpitaux musulmans créés en temps de guerre, tant en Espagne, pendant la guerre civile qui impliqua des troupes marocaines (Moreras et Tarrés), qu'en France, puisque le cimetière de Bobigny fut créé après la Première Guerre mondiale - durant laquelle nombreux furent les morts musulmans - (El Alaoui). Les références à I'histoire coloniale, propre à chacun des pays, sont contrastées et parfois très anciennes : pour obtenir aujourd'hui des conditions spécifiques d'inhumation, certains groupes musulmans d'Espagne se réfèrent à une colonisation (musulmane) de l'Espagne qui s'acheva à la fin du XVe siècle.

Tous les migrants ne sont pas enterrés dans le pays où ils résident. Les restes d'une partie d'entre eux, très variable selon les groupes sociaux et nationaux ${ }^{2}$, sont ramenés vers le lieu d'origine. Pays d'accueil et de départ doivent donc également faire face aux transferts des corps des migrants d'un État à l'autre en encadrant leurs déplacements (Chaïb, 2000 ; Lestage, 2008) et les processus commerciaux qui les permettent, comme je

2 Selon Gaudart et Taussig (2009), cités par El Alaoui (dans ce dossier), $85 \%$ des corps des musulmans de France sont rapatriés en 2007 alors que 95 \% l'étaient en 1997. Pour Lestage (2008), ce sont $10 \%$ environ des corps des migrants mexicains qui seraient rapatriés au Mexique en 2006. 
le détaille dans mon article portant sur la chaîne entrepreneuriale de l'industrie mexicaine du transfert funéraire international, niche économique en plein développement, occupée par des acteurs migrants et non migrants qui ménagent les croyances et les pratiques des pays d'origine et d'accueil afin de satisfaire au mieux leurs clients.

\section{Le " retour " post-mortem des migrants, un élément du projet migratoire}

Les auteurs de la plupart des travaux sur la mort en migration affirment que le rapatriement du cadavre, "éternel retour à l'origine "(Chaïb, 2000 : 57), est une caractéristique des migrants qu'ils étudient ; tous y voient des raisons d'ordre historique, religieux ou mythique. Pour Chaïb (2000), ce sont les habitants du pourtour méditerranéen qui auraient eu des coutumes spécifiques liées au transfert des défunts, pratiques contraires à ce que décrète l'Islam pour lequel le mort n'appartient pas à la société des vivants, mais à Dieu, et doit donc lui être remis dès que possible (Aggoun, 2006). D'après Felix (2011), c'est l'imaginaire national mexicain, nourri de références à la mort, qui orienterait le choix du retour du corps au pays. Selon Pourrette, c'est la seule façon " d'accéder au statut d'ancêtre " pour les Guadeloupéens (Pourrette, 2002 : 54).

Cette récurrence du désir du " retour " post-mortem que tous les auteurs analysent en utilisant des clés d'interprétation locales, ne doit-elle pas être considérée comme partie intégrante du projet migratoire et comme une constante de celui-ci ? En effet, tous les migrants partent pour revenir un jour, du moins le croient-ils et le disent-ils. Seule une partie d'entre eux accomplira ce souhait, les autres l'abandonneront. II en est de même pour le retour post-mortem : certains l'abandonneront ; $d$ 'autres feront en sorte - eux ou leurs familles - de réaliser ce projet après leur mort et le justifieront alors en alléguant I'histoire, la religion ou I'appartenance à la lignée ou à la nation.

Tant la réalisation du retour post-mortem que sa justification sont aléatoires. À la lecture des travaux déjà cités, on constate que les rapatriements des corps ne répondent pas à des logiques générales, mais à une série de variables qui imbriquent les histoires personnelles des migrants et les rapports qu'ils entretiennent avec les groupes sociaux $d^{\prime}$ 'origine et $d$ 'accueil. Parmi ces variables, on retiendra le temps passé en migration par le migrant et/ou sa famille et la taille du groupe immigré en un même lieu : I'article sur les Guinéens et les Bangladais au Portugal donne à voir des populations de migrants dans lesquelles les liens au lieu et au groupe d'origine ne se sont pas distendus. On retiendra également d'autres variables comme les politiques migratoires des pays d'accueil et d'origine qui favorisent ou compliquent le transfert du défunt, ainsi que les normes sociales en vigueur dans le groupe social de rattachement qui prescrivent les règles d'une " bonne mort ".

\section{Les représentations sociales de la « bonne mort "}

Car le rapatriement des corps des migrants relève de conceptions de la " bonne mort " qui varient selon les époques et les groupes sociaux. II ne faut pas confondre " bonne mort " et " belle mort " (Thomas, $1985:$ 132). La " belle mort " concerne exclusivement le mourir alors que la " bonne mort " se réfère autant, sinon plus, à ce qu'il advient après la mort, c'est-à-dire son traitement (sépulture, rituels funéraires). Tous ces évènements sont normés. Ils doivent se produire, idéalement, dans des conditions définies par le groupe social et, souvent, par le dogme religieux. Dans les sociétés occidentales et chrétiennes, une des règles de la " bonne mort " consiste à éviter la mort en solitaire et donc à accompagner le moribond, puis le défunt, jusqu'à ce que l'on soit assuré de l'avoir conduit dans I'au-delà ; des systèmes structurés s'instaurent parfois, telles les " confréries de la bonne mort " à Montréal du XVIle au XIXe siècle (Caulier, 1997), précisément destinées à 
des migrants n'ayant pas de famille en ville pour effectuer les rites funéraires adéquats. Ailleurs, la mort organise la vie et la société comme dans le célèbre cas des Merina à Madagascar, décrit et analysé par Bloch (1971). Chez les Merina, chaque personne est membre d'un groupe en fonction de son rattachement à un même tombeau qui accueille les " morts au loin ". C'est la place dans le tombeau familial et la manipulation des corps des défunts qui constitue le groupement. "Mettre les morts en place " (placing the dead) (Bloch, 1971) représente une activité fondamentale qui a pour objet d'organiser la parenté et le groupe social.

Si la " bonne mort " n'est pas au fondement de l'organisation de toute société, elle reste, pour tous, migrants ou pas, la façon de remettre de l'ordre dans la vie sociale et de réaffirmer cet ordre après la disparition de l'un de ses membres (Déchaux, 1997 : 33). Les migrants et leurs familles sont amenés à redonner du sens aux pratiques et aux rites, à les " resignifier ", mais aussi à reformuler les règles de la " bonne mort " en tenant compte à la fois des sociétés d'origine et d'accueil : par exemple, mourir du VIH interdit l'accès au cimetière familial sur l'île pour les Guadeloupéens de métropole et seule la crémation est en mesure de rétablir l'ordre social troublé par une telle maladie (Pourrette, 2002 : 57-58).

Dans leur article, Clara Saravia et José Mapril analysent les discours et pratiques autour du traitement des défunts dans deux groupes de migrants au Portugal, les Bangladais, émigrés peu nombreux arrivés récemment, et les Guinéens, flux plus ancien issu d'une ex-colonie portugaise. Ils constatent que les gestes et les rites sont destinés à créer les conditions d'une "bonne mort " dans les deux groupes de migrants, autrement dit de garantir la continuité des relations entre les vivants où qu'ils résident ; entre les vivants et les morts comme seul et même groupe; entre eux tous et les lieux qui comptent pour eux (ceux des sépultures notamment). Leur article résume en une seule notion les arguments variés avancés pour rapatrier les défunts par d'autres auteurs. Au fond, qu'ils soient d'ordre historique, religieux ou mythique, tous correspondent aux représentations sociales de la " bonne mort " dans les groupes de migrants.

\section{Le brouillage du dualisme immigration-émigration}

Même si les travaux actuels sur la mort en migration évoquent les pratiques multisituées des migrants et des non-migrants, ils suivent surtout les deux options principales, mais pas exclusives, adoptées par les migrants : laisser le défunt sur place, ou le ramener au pays, sous une forme ou une autre. D'un côté, ces travaux s'attachent à l'étude des espaces funéraires des pays d'accueil et posent la question du point de vue de l'immigration : le rapport à l'État d'accueil, les politiques publiques, l'articulation laïcité-religion, la patrimonialisation et les lieux de la mémoire migratoire. De l'autre, ils se consacrent à l'étude des transferts des restes ou des possessions des migrants défunts vers le pays d'origine et posent la question du point de vue de l'émigration : I'appartenance au lignage et à la communauté d'origine, le rapport à l'État de départ, le statut national de l'émigré.

Cette deuxième approche prend place dans une réflexion sur la mobilité des restes humains. Or, une telle mobilité n'a rien d'exceptionnel. Reliques, cadavres, cendres et poussières se déplacent et sont déplacés (Esquerre et Truc, 2011) et les défunts peuvent être présents en plusieurs lieux, bien plus aisément que les vivants. Ils le sont au sens propre puisqu'on peut les fragmenter, ou bien d'un point de vue symbolique, grâce à leurs photos ou celles de leurs tombes (Lestage, 2009 : 451) ou grâce à des objets les représentant, comme la " valise du mort ", ramenée dans le lieu d'origine du migrant, en Guinée-Bissau, alors que son corps reste dans le pays d'accueil, au Portugal (Petit, 2002 ; Saravia et Mapril, dans ce dossier). On ne peut plus évoquer alors une " double absence " (Sayad, 1999), mais une double ou multiple présence. 
Sur ce point, les auteurs du dossier ne parlent pas d'une seule voix : alors que Moreras et Tarrés confèrent une place prépondérante à la dualité émigration-immigration, Saravia et Mapril insistent sur le brouillage de I'opposition ici/là-bas et sur l'utilisation, par les migrants, de la mort d'un des leurs comme un élément fédérateur et régénérateur pour le groupe social dans son entier. Peut-on dire, aujourd'hui encore, que la mort des migrants est " inclassable " ou " déplacée ", "indécente " ou "incongrue " (Sayad, 2000 : 9) comme le suggèrent Moreras et Tarrés au début de leur article ? Ou la mort n'est-elle qu'une démonstration de plus de la capacité des migrants vivants à ajuster leurs pratiques à la mobilité géographique et socio-culturelle contemporaine, comme le laissent entendre Saravia et Mapril ? Cette divergence n'enlève rien à l'unité d'un dossier dont les cas de figure exposés, forcément limités, pourraient s'élargir à d'autres contextes nationaux, à d'autres transferts de corps ou d'objets, à d'autres pratiques funéraires, renouvelant ainsi la réflexion sur les migrations à partir de la mobilité des morts, et non plus des vivants.

\section{Références bibliographiques}

Aggoun Atmane (2006) Les musulmans face à la mort, Paris, Vuibert, 156 p.

Ariès Philippe (1975) Essai sur I'histoire de la mort en Occident. Du Moyen Âge à nos jours, Paris, Points-Seuil, 226 p.

Attias-Donfut Claudine et François-Charles Wolff (2005) Le lieu d'enterrement des personnes nées hors de France, Population, 5 (60), pp. 813-816, [en ligne]. DOI : 10.3917/ popu.505.0813

Baudry Patrick (1999) La place des morts. Enjeux et rites, Paris, Armand Colin, 206 p.

Berthod Marc-Antoine (2006) Expérience migratoire et identité dans la mort transnationale : les défunts portoricains rapatriés de New York, Revue Canadienne des Études Latino-américaines et Caraïbes, 61 (31), pp. 145-168.

Bloch Maurice (1971) Placing the Dead. Tombs, Ancestral Villages and Kinship Organization in Madagascar, London and New York, Seminar Press, 241 p.

Bondaz Julien (2011) Le cadavre et le politique. La vie sociale des restes humains, La vie des idées, [en ligne]. URL : http://www.laviedesidees.fr/Le-cadavre-et-le-politique.html

Branquinho Pequeno António (1983) Les morts voyagent aussi... " Le dernier retour " de l'immigré portugais, Esprit, novembre, pp. 153-156.

Caulier Brigitte (1997) Pour une bonne mort : les confréries et la mort à Montréal (XVIleXIXe siècles), Frontières, 5 (1), pp. 14-18.

Chaïb Yassine (2000) L'émigré et la mort, Aix-en-Provence, CIDIM-Edisud, 255 p.

Douglass William (1969) Death in Murelaga. Funeral Ritual in a Spanish Basque Village, Seattle, University of Washington Press, 244 p.

Esquerre Arnaud et Gérome Truc (2011) Les morts, leurs lieux et leurs liens, Raisons politiques, 41 (1), pp. 5-11. 
Fall Khadiyaoulah et Mamadou Ndongo Dime (2011) La mort musulmane en contexte d'immigration et d'islam minoritaire. Enjeux religieux, culturels, identitaires et espaces de négociation, Québec, Presses de I'Université Laval, 238 p.

Godard Bernard et Sylvie Taussig (2007) Les Musulmans en France. Courants, institutions, communautés : un état des lieux, Paris, Hachette Littératures, 464 p.

Hertz Robert (1928) Contribution à une analyse des rites funéraires, in Robert Hertz, Mélanges de sociologie religieuse et de folklore, Paris, Alcan (1ère édition :1907).

Lestage Françoise (2009) Vivants et morts dans les migrations mexicaines : un système de relations inscrit dans la mobilité, in Virginie Baby-Collin, Geneviève Cortes, Laurent Faret, Hélène Guétat-Bernard Éds., Migrants des Suds. Acteurs et trajectoires de la mobilité internationale, IRD, Université de Montpellier, Presses Universitaires du Mirail (PUM), pp. 431-452.

Lestage Françoise (2008) Apuntes relativos a la repatriación de los cuerpos de los mexicanos fallecidos en Estados Unidos, Migraciones Internacionales, 4 (4), pp. 217-228.

Petit Agathe (2002) La mort au loin. Les pratiques funéraires des migrants africains en France, Thèse d'ethnologie et d'anthropologie sociale, Marseille, EHESS, sous la direction d'André Mary.

Pourette Dolorès (2002) Pourquoi les migrants guadeloupéens veulent-ils être inhumés dans leur île?, Hommes et Migrations, 1237, pp. 54-61.

Sayad Abdelmalek (1999) La double absence. Des illusions de l'émigré aux souffrances de I'immigré, Paris, Seuil, 438 p.

Sayad Abdelmalek (2000) Préface, in Chaïb Yassine, L'émigré et la mort, Aix-en-Provence, CIDIM-Edisud, pp. 5-18.

Trong Hien Dinh (1990) Rythmes des vivants, mémoires des morts. Espace temps, rituels du culte des ancêtres, Hommes et Migrations, 1134, pp. 19-21. 